\title{
The Influence of Solvent, Host, and Phenological Stage on the Yield, Chemical Composition, and Antidiabetic and Antioxidant Properties of Phragmanthera capitata (Sprengel) S. Balle
}

\author{
Césaire Feudjio $\left(\mathbb{D},{ }^{1,2}\right.$ Muhammad Arfat Yameen $\mathbb{D}^{1},{ }^{2}$ Guy Sedar Singor Njateng $\left(\mathbb{D},{ }^{1}\right.$ \\ Muhammed Ahsan Khan $\mathbb{D D}^{2}{ }^{2}$ Stephen Lacmata Tamekou $\mathbb{D}^{1},{ }^{1}$ James Deke Simo Mpetga $\left(\mathbb{D},{ }^{3}\right.$ \\ and Jules-Roger Kuiate (iD \\ ${ }^{1}$ Research Unit of Microbiology and Antimicrobial Substances, Faculty of Science, Department of Biochemistry, \\ University of Dschang, P.O. Box 67, Dschang, Cameroon \\ ${ }^{2}$ COMSATS University of Information Technology, Abbottabad Campus, Islamabad 22060, Pakistan \\ ${ }^{3}$ Natural Products Chemistry Research Unit, Faculty of Science, Department of Chemistry, University of Dschang, P.O. Box 67, \\ Dschang, Cameroon
}

Correspondence should be addressed to Jules-Roger Kuiate; jrkuiate@yahoo.com

Received 20 July 2020; Revised 24 September 2020; Accepted 19 October 2020; Published 19 November 2020

Academic Editor: Luigi Milella

Copyright (c) 2020 Césaire Feudjio et al. This is an open access article distributed under the Creative Commons Attribution License, which permits unrestricted use, distribution, and reproduction in any medium, provided the original work is properly cited.

\begin{abstract}
Phragmanthera capitata was reported to possess many biological properties making it a good candidate for the formulation of a phytomedicine with multiple effects. In this work, we studied some factors likely to modify these therapeutic properties with the aim to contribute to its standardization as an improved traditional medicine. P. capitata parasitizing Persea americana, Psidium guajava, and Podocarpus mannii were harvested at three phenological stages (vegetative, flowering, and fruiting stages). The extracts were prepared by maceration in n-hexane, ethyl acetate, ethanol, methanol, and distilled water. The total phenolic, flavonoid, flavonol, and tannin contents were measured using appropriate methods. The antioxidant potential of extracts was investigated using TAC, DPPH scavenging, and FRAP methods. The $\alpha$-amylase and $\alpha$-glucosidase inhibitory activities of extracts were determined using enzymatic methods. The ethyl acetate extracts with the best phenolic content were subjected to HPLC analysis. The extraction yields were higher with methanol. The ethyl acetate extract of $P$. capitata harvested from $P$. guajava showed a stable HPLC profile during the development of the plant, while extracts from the plant collected from $P$. americana and $P$. mannii showed both qualitative and quantitative variations according to phonological stages of the plant. The inhibition of $\alpha$-amylase was more pronounced for P. capitata harvested from P. guajava, decreasing during flowering and fruiting, while inhibition of $\alpha$-glucosidase was not influenced by the phenological stage and the host of the plant. The $\alpha$-amylase inhibitors were better extracted by ethyl acetate and those of $\alpha$-glucosidase by ethanol or methanol. The phenolic contents and antioxidant properties of the extracts were influenced by the phenological stage of $P$. capitata and its hosts. These results suggest that it is preferable to harvest $P$. capitata during flowering or during fruiting stages on any host. None of the used solvents permitted an optimal extraction of active principles form $P$. capitata, suggesting that the mixture of solvents must be considered in further studies.
\end{abstract}

\section{Introduction}

Phragmanthera capitata is a mistletoe plant belonging to the Loranthaceae family. It is a mandatory hemiparasite that attaches and enters the stems and branches of its host tree through a haustorium $[1,2]$. This plant is widely distributed in Cameroon and in some other African countries. It is characterized by yellow flowers with red apex [3]. It can grow on various plants, including Persea americana, Psidium guajava, Citrus sinensis, Podocarpus mannii, Hevea brasiliensis, Spondias mangifera, Garcinia kola, Manniophyton fulvum, Theobroma cacao, and Citrus spp. [4]. In Cameroon, 
leaf, stem, or whole plant is claimed to cure up to 38 diseases, including infectious diseases, nerve pain, cancers, and cardiac disorders, but mostly used to cure hypertension and diabetes $[5,6]$.

Previous scientific reports demonstrated that extracts from this plant have many biological activities, such as antioxidant, anti-inflammatory [7], hematopoietic potentiating [8], antibacterial, antifungal [9], antisecretory, gastroprotective, and antiulcer [10], and that they also enhance steroidogenetic and spermatogenetic activities [11]. In a previous research work in our laboratory, we demonstrated that the administration of Phragmanthera capitata aqueous extract to diabetic rats significantly decreased hyperglycaemia at the dose used by the herbal medicine doctors. It showed hypolipidemic and immunomodulatory effects as well [12]. Furthermore, the water extract from P. capitata showed no toxic reactions, no change in behavior, and no mortality at a dose of $3000 \mathrm{mg} / \mathrm{kg}$ and $5000 \mathrm{mg} / \mathrm{kg}$ in mice and Wistar rats [7, 13], while acetone, methanol, ethanol, and water extracts showed not toxic (lethal concentration $>1 \mathrm{mg} / \mathrm{mL}$ ) in Brine shrimp hatchability assay. Hence, the solvent extracts from this plant can be further explored for the development of plant-based pharmaceuticals drugs [14].

This parasitic plant is a real problem for crops. Thus, several methods using synthetic herbicides have been developed to eradicate it without much success. Chemical control has side effects on the environmental pollution and food health [15]. Given its multiple biological properties, this plant could be exploited as a phytomedicine and deprived of its host for this reason. In addition, one of the major problems faced by the phytomedicine is the unavailability of rigid quality control profiles for herbal materials and their formulations [16]. Moreover, the solvent effect is an important parameter for phytomedicine standardization, allowing selection of a suitable solvent for herbal processing. The studies of Ohikhena et al. $[17,18]$ showed the influence of the solvent on the antioxidant and antidiabetic activities of the extract of leaves from $P$. capitata growing on rubber tree. The standardization of extracts is necessary to guarantee the desired properties at all times. Indeed, plant extract composition and biological activities may vary due to climatic differences, phenological stages, soil composition, and environmental stress. Additionally, for some plants, chemo varieties and chemo cultivars may exist [14], making it difficult for untrained people to discriminate against them. The harvesting, drying, storage, transportation, and processing methods may also influence herbal quality and pharmacological properties [19].

The present work aimed to study the influence of certain factors, in particular, the phenology of the plant, the extraction solvent, and the nature of the host of $P$. capitata on the phytochemistry, antioxidant, and antidiabetic properties of the extracts of this parasitic plant. This study should generate preliminary data for a possible standardization of extracts of $P$. capitata as an antioxidant phytomedicine to fight oxidative stress associated with the many disease conditions that are currently being managed locally with the plant.

\section{Materials and Methods}

2.1. Processing of Plant Samples. Phragmanthera capitata (Sprengel) S. Balle was harvested in Bamendou village, Menoua division of the west region of Cameroon. It was recommended by a traditional healer working in the locality. The whole plant sample was harvested in the morning, between 8 and $10 \mathrm{am}$, from each of three different plant hosts, namely, Persea americana, Psidium guajava, and Podocarpus mannii, and at three different phenological stages: during vegetative (January 2018), during flowering (March 2018), and during fruiting (April 2018). P. capitata and its selected host plants were authentified at the Cameroon National Herbarium in Yaoundé, by comparison to registered voucher specimens (Table 1). Each plant sample was cleaned and dried for 3 weeks in a ventilated room at ambient temperature $\left(22 \pm 2^{\circ} \mathrm{C}\right)$. The dried plant material was ground and stored in plastic bags until extraction. The study was conducted according to the Guidelines on the Conservation of Medicinal Plants of the WHO [20].

2.2. Preparation of Extracts. Five different solvents of increasing polarity were individually used for the preparation of extracts, including n-hexane, ethyl acetate, ethanol, methanol, and water. One hundred grams $(100 \mathrm{~g})$ of each powder was macerated in $500 \mathrm{~mL}$ in the corresponding solvent for $48 \mathrm{~h}$ under mechanical stirring. The resulting mixture was vacuum filtered through Whatman filter paper $\mathrm{N}$ 1. The obtained organic filtrates (except aqueous filtrate) were evaporated under low pressure using a Buchi R210 evaporator at $40^{\circ} \mathrm{C}$. The resulting extracts were subjected to a $40^{\circ} \mathrm{C}$ drying in an oven for 24 hours to remove the residual solvent. The aqueous filtrates aliquots of $20 \mathrm{ml}$ were dried in an oven at $40^{\circ} \mathrm{C}$ for 5 days in stainless plates $(30 \mathrm{~cm}$ diameter).

\subsection{Evaluation of Antidiabetic Activity of P. capitata Extracts}

2.3.1. Alpha-Amylase Inhibitory Assay. The $\alpha$-amylase inhibitory effects of extracts were determined using the DNSA method as described by Kazeem et al. [21] adapted for 96well microplates. The DNSA reagent was freshly prepared by dissolving $1 \mathrm{~g}$ of DNSA and $30 \mathrm{~g}$ of potassium sodium tartrate in $40 \mathrm{~mL}$ of distilled water, after which $20 \mu \mathrm{L}$ of $\mathrm{NaOH}(2 \mathrm{M})$ was added, and then, the total volume was adjusted to $100 \mathrm{~mL}$ with distilled water. This was performed under magnetic stirring on a $40^{\circ} \mathrm{C}$ hot plate. Aliquots of $20 \mu \mathrm{L}$ of sodium phosphate buffer $(0.02 \mathrm{M}, \mathrm{pH} 6.9)$ and $\alpha$-amylase solution $(0.5 \mathrm{mg} / \mathrm{mL})$ were added to $20 \mu \mathrm{L}$ of varying concentrations $(3.25-800 \mu \mathrm{g} / \mathrm{mL})$ of the extract or acarbose $(2 \mathrm{mg} / \mathrm{mL}$ in DMSO $1 \%)$. The microplate was preincubated at $37^{\circ} \mathrm{C}$ for $5 \mathrm{~min}$, after which $20 \mu \mathrm{L}$ of $1 \%$ starch solution in sodium phosphate buffer $(0.02 \mathrm{M}, \mathrm{pH} 6.9)$ was added and then further incubated at $37^{\circ} \mathrm{C}$ for $30 \mathrm{~min}$. The reaction mixture was stopped by adding $20 \mu \mathrm{L}$ of DNSA reagent. The microplate was then placed in a waterbath at $85^{\circ} \mathrm{C}$ for 10 min to allow the reaction between DNSA and maltose from the hydrolysis of starch. This reaction was then stopped by placing the microplate in an ice waterbath for 
TABLE 1: Information about the plant material used in the study.

\begin{tabular}{lcc}
\hline Species & Role & Registered voucher code \\
\hline Phragmanthera capitata (Sprengel) S. Balle & Studied plant & $24673 /$ SRF/CAM \\
Persea americana Mill. (Lauraceae) & Host 1 & $57756 / \mathrm{HNC}$ \\
Psidium guajava Linn. (Myrtaceae) & Host 2 & $2884 / \mathrm{SRF} / \mathrm{CAM}$ \\
Podocarpus mannii Hook. f (Podocarpaceae) & Host 3 & $5088 / \mathrm{HNC}$ \\
\hline
\end{tabular}

SRF, Cameroon forest reserve society; HNC, Cameroon national herbarium.

3 min. The reaction mixture was diluted with $100 \mu \mathrm{L}$ of distilled water. A negative control was prepared using the same procedure replacing the extract with DMSO solution (1\%). The absorbances were read at $540 \mathrm{~nm}$ using an Elisa Bio-Rad PR 4100 microplate reader. The $\alpha$-amylase inhibitory activity was calculated as percentage inhibition of starch hydrolysis:

$\%$ inhibition $=\left[\frac{\text { Absorbance }_{\text {control }}-\text { absorbance }_{\text {test }}}{\text { Absorbance }_{\text {control }}}\right] \times 100$.

Concentrations of extracts or acarbose resulting in 50\% inhibition of enzyme activity ( $\mathrm{IC}_{50}$ in $\mu \mathrm{g} / \mathrm{mL}$ ) were obtained by applying regression analysis.

2.3.2. Alpha-Glucosidase Inhibitory Assay. The $\alpha$-glucosidase inhibitory activities of extracts were determined according to the method described by Bljajić et al. [22] adapted for 96-well microplates, using $\alpha$-glucosidase from Saccharomyces cerevisiae type I (Sigma-Aldrich, US) and p-nitrophenylglucopyranoside (pNPG, Sigma-Aldrich, US) as the substrate. The solution of $\alpha$-glucosidase was prepared at $0.5 \mathrm{U} / \mathrm{mL}$ by dilution of a $9 \mathrm{U} / \mathrm{mL}$ stock solution. The substrate solution was prepared by dissolving $3 \mathrm{mg}$ of pNPG in $1 \mathrm{~mL}$ of phosphate buffer $(0.07 \mathrm{M}, \mathrm{pH} 6.8)$. Aliquots of $62 \mu \mathrm{L}$ of phosphate buffer and $12 \mu \mathrm{L}$ of $\alpha$-glucosidase solution were added to $10 \mu \mathrm{L}$ of varying concentrations $(3.25-800 \mu \mathrm{g} / \mathrm{mL})$ of the extract or acarbose $(2 \mathrm{mg} / \mathrm{mL}$ in DMSO $1 \%$ ). The microplate was preincubated at $37^{\circ} \mathrm{C}$ for $5 \mathrm{~min}$. Then, $16 \mu \mathrm{L}$ of substrate (pNPG) solution was added to start the reaction. The microplate was homogenized and incubated at $37^{\circ} \mathrm{C}$ for $30 \mathrm{~min}$. A negative control was prepared using the same procedure replacing the extract with DMSO solution (1\%). The absorbances were read at $405 \mathrm{~nm}$ using an ELx800 microplate reader. Percentage of enzyme inhibition by the extract (or acarbose) was calculated using the following equation:

$\%$ inhibition $=100-\left[\frac{\text { Absorbance }_{30 \text { min }}-\text { absorbance }_{5 \mathrm{~min}}}{\text { Absorbance }_{\text {control }}}\right] \times 100$.

Concentrations of extracts or acarbose necessary to inhibit $50 \%$ activity of the enzyme $\left(\mathrm{IC}_{50}\right)$ were obtained by applying regression analysis.

\subsection{Chemical Characterization and Antioxidant Properties of} P. capitata Extracts. Five known compounds were isolated and characterized in ethyl acetate extracts, and this is described in the Supplementary material. The other aspect of chemical characterization of different extracts comprised total phenol (TPC), total flavonoids (TFC), total flavonols (TfnC), and total tannin content (TTC) determinations. For this purpose, a stock solution of each plant extract was prepared at a concentration of $4 \mathrm{mg} / \mathrm{mL}$ in DMSO $1 \%$. Prior to this, the ethyl acetate extract that showed the best antioxidant activity was submitted to HPLC for compound identification.

2.4.1. HPLC Profile of Ethyl Acetate Extracts. HPLC analyses were performed on different ethyl acetate extracts. It was used to determine the gallic acid, quercetin, rutin, and tannic acid proportions in the ethyl acetate plant extracts. The HPLC system used (Shimadzu 20 AD, Japan) consists of an ultraviolet detector, a binary pump, a $20 \mu \mathrm{L}$ injection loop, and Shim-Pack GIST C18 $(150 \mathrm{~mm} \times 4,6 \mathrm{~mm}$ i.d. $\times 5 \mu \mathrm{m})$ column. The mobile phase consisted of two solvents, namely, acetonitrile, HPLC grade (solvent A), and formic acid ( $1 \%$ in deionized water), HPLC grade (solvent $\mathrm{B}$ ). The elution gradient was established as follows: $0 \mathrm{~min}$ ( $5 \% \mathrm{~A}$ and $95 \% \mathrm{~B})$, 0-21 min (5-20\% A and 95-80\% B), 21-30 min (20-25\% A and $80-75 \% \mathrm{~B}), 30-32 \mathrm{~min}(25-100 \% \mathrm{~A}$ and $75-0 \% \mathrm{~B})$, $32-39 \min (100-100 \% \mathrm{~A}$ and $0 \% \mathrm{~B}), 39-40 \mathrm{~min}(100-5 \% \mathrm{~A}$ and $0-95 \% \mathrm{~B})$, and $40-45 \mathrm{~min}(5-5 \% \mathrm{~A}$ and $95 \% \mathrm{~B})$. The flow rate of this mobile phase was $0.7 \mathrm{~mL} / \mathrm{min}$.

The extracts and standard phenolic compounds were prepared at a concentration of $10 \mathrm{mg} / \mathrm{ml}$ in acetonitrile (HPLC grade). Each solution was then diluted to $100 \mu \mathrm{g} / \mathrm{mL}$ and then filtered with a syringe filter (Corning, $0.45 \mu \mathrm{m}$ ) to obtain the solutions to be analyzed. The mixture of standards phenolic compounds was prepared immediately, and $20 \mu \mathrm{L}$ of mixture solution was injected into HPLC to get the profile of standard. After column cleaning, $20 \mu \mathrm{L}$ of each plant extract was then injected into the HPLC system to determine its chemical profile. The proportion of gallic acid, quercetin, rutin, and tannic acid in the ethyl acetate plant extracts was determined by comparing the peak area (AUC) of the compound identified in the profile of the extract to its area in the profile of the standard [23].

2.4.2. Total Phenolic Content Determination. The total phenolic content (TPC) of extracts was determined using the Folin-Ciocalteu colorimetric method as described by Horszwald and Andlauer [24] adapted for 96-well microplates. A calibration curve was established with gallic acid $(0-50 \mu \mathrm{g} / \mathrm{mL})$. The Folin-Ciocalteu reagent $(90 \mu \mathrm{L})$ (Sigma) diluted 10 times with distilled water was mixed to $20 \mu \mathrm{L}$ of the extract or gallic acid solution. A $7 \% \mathrm{Na}_{2} \mathrm{CO}_{3}$ solution 
(90 $\mu \mathrm{L}$ ) was then added followed by thorough mixing and incubation in the dark at room temperature $\left(22 \pm 2^{\circ} \mathrm{C}\right)$ for $60 \mathrm{~min}$. The blank solution was prepared as above, replacing the extract or gallic acid with distilled water. The absorbance was read at $765 \mathrm{~nm}$ using an ELx800 microplate reader. The amount of phenolic content was derived from the calibration curve and expressed as mg equivalent of gallic acid per gram of dry extract (mg GAE/g).

2.4.3. Total Flavonoids Content. The total flavonoids content (TFC) was assayed using the $\mathrm{AlCl}_{3}$ method as described by Laloo and Sahu [25] adapted for 96-well microplates. A calibration curve was established with rutin $(0-100 \mu \mathrm{g} / \mathrm{mL})$. The $\mathrm{AlCl}_{3}$ solution $(10 \mu \mathrm{L})(10 \%$ in distilled water $)$ was added to $20 \mu \mathrm{L}$ of the extract or rutin solution. After $5 \mathrm{~min}, 10 \mu \mathrm{l}$ of sodium acetate solution ( $1 \%$ in distilled water) was added to each well. The total volumes were adjusted to $200 \mu \mathrm{L}$ with distilled water, followed by thorough mixing and incubation in the dark at room temperature $\left(22 \pm 2^{\circ} \mathrm{C}\right)$ for $15 \mathrm{~min}$. The blank solution was prepared as above, replacing the extract or rutin by distilled water. The absorbance was read at $415 \mathrm{~nm}$ using an ELx800 microplate reader. The amount of flavonoids in plant extracts was derived from the calibration curve. Flavonoid content was expressed in mg equivalents of rutin per g of dry extract (mg RUE/g).

2.4.4. Total Flavonols Content. The total flavonols content (TfnC) was assayed using the $\mathrm{AlCl}_{3}$ method according to Awah et al. [26] adapted for a 96-well microplate format. The calibration curve was established with rutin $(0-100 \mu \mathrm{g} / \mathrm{mL})$. Briefly, $40 \mu \mathrm{L}$ of the plant extract or rutin solution was mixed with $40 \mu \mathrm{L} \mathrm{AlCl}_{3}(20 \mathrm{mg} / \mathrm{mL}$ in ethanol, HPLC grid). After $5 \mathrm{~min}, 120 \mu \mathrm{L}$ of sodium acetate solution $(50 \mathrm{mg} / \mathrm{mL}$ in ethanol, HPLC grade) was added to each well. The blank was prepared by replacing the extract or rutin with distilled water. The absorbance of each mixture was read at $440 \mathrm{~nm}$ after $2 \mathrm{~h} 30 \mathrm{~min}$ of incubation in the dark and at room temperature, using an ELx800 microplate reader. The amount of flavonols in plant extracts was derived from the calibration curve and expressed in mg equivalents of rutin per gram of dry extract (mg RUE/g).

2.4.5. Total Tannin Contents. Total tannin contents (TTC) were assayed according to the Gupta and Verma [27] protocol adapted for 96-well microplates. The calibration curve was established with tannic acid $(0-100 \mu \mathrm{g} / \mathrm{mL})$. Briefly, $20 \mu \mathrm{L}$ of $0.1 \mathrm{M} \mathrm{FeCl}_{3}$ (in $0.1 \mathrm{~N} \mathrm{HCl}$ ) was added to $20 \mu \mathrm{L}$ of the plant extract or tannic acid, followed immediately by addition of $20 \mu \mathrm{L}$ of $0.008 \mathrm{M}$ of $\mathrm{K}_{3} \mathrm{Fe}(\mathrm{CN})_{6}$ (in distilled water). The volumes were adjusted to $200 \mu \mathrm{L}$ with $140 \mu \mathrm{L}$ of distilled water followed by thorough mixing and incubation in the dark room temperature for $10 \mathrm{~min}$ to allow color to become more stable. The blank was prepared by replacing the extract or tannic acid with distilled water. The absorbances were read at $720 \mathrm{~nm}$ using an ELx800 microplate reader. The amount of tannin in the plant extracts was derived from the calibration curve and expressed as mg of tannic acid equivalents per $g$ of dry extract (mg TAE/g).

(1) Determination of Antioxidant Activities. Three methods were used to determine the antioxidant potential of various extracts: total antioxidant capacity (TAC), ferric reducing antioxidant power (FRAP), and 2,2-diphenyl-1-picrylhydrazyl (DPPH) scavenging activity. For this purpose, a stock solution of each plant extract was prepared at a concentration of $2 \mathrm{mg} / \mathrm{mL}$ in DMSO 1\%. All experiments were carried out in triplicate.

(2) Determination of the Total Antioxidant Capacity. The total antioxidant capacity (TAC) of extracts was measured with the phosphomolybdenum method according to Hossain and Shah [28] protocol adapted for 96-well microplates. The phosphomolybdate reagent solution was prepared freshly by mixing $50 \mathrm{~mL}$ of $\mathrm{H}_{2} \mathrm{SO}_{4}(0.6 \mathrm{M}), 50 \mathrm{~mL}$ of $\mathrm{Na}_{3} \mathrm{PO}_{4}$ $(28 \mathrm{mM})$, and $50 \mathrm{~mL}$ of $\left(\mathrm{NH}_{4}\right)_{6} \mathrm{Mo}_{7} \mathrm{O}_{24} 4 \mathrm{H}_{2} \mathrm{O}(4 \mathrm{mM})$. The calibration curve was established with ascorbic acid $(0-50 \mu \mathrm{g} / \mathrm{mL})$. The phosphomolybdate reagent $(150 \mu \mathrm{L})$ was added to $15 \mu \mathrm{L}$ of extracts or ascorbic acid. The blanks were constituted by mixing $150 \mu \mathrm{L}$ of phosphomolybdate reagent solution and $15 \mu \mathrm{L}$ of DMSO. The microplate was incubated in a waterbath at $80^{\circ} \mathrm{C}$ for $90 \mathrm{~min}$, then cooled down to room temperature. The absorbance was read at $695 \mathrm{~nm}$ using an ELx800 microplate reader. The total antioxidant capacity (TAC) of plant extracts was derived from the calibration curve of ascorbic acid and expressed as equivalent of ascorbic acid.

(3) Ferric Reducing Antioxidant Power. The method of Vijayalakshmi and Ruckmani [29] using $\mathrm{K}_{3} \mathrm{Fe}(\mathrm{CN})_{6}$ adapted on 96-well microplates was used. The calibration curve was established with ascorbic acid $(0-100 \mu \mathrm{g} / \mathrm{mL})$. Volumes of $50 \mu \mathrm{L}$ of phosphate buffer $(20 \mathrm{mM}, \mathrm{pH} 6.6)$ and potassium ferricyanide solution $(1 \% \mathrm{w} / \mathrm{w})$ were, respectively, added to $20 \mu \mathrm{L}$ of the extract or ascorbic acid. The mixtures were incubated in a waterbath at $50^{\circ} \mathrm{C}$ for 20 minutes. The microplate was removed from the waterbath, and $50 \mu \mathrm{L}$ of trichloroacetic acid $(10 \% \mathrm{w} / \mathrm{w})$ was added into each well, and then left standing for $60 \mathrm{~min}$. The supernatant $(10 \mu \mathrm{L})$ was diluted with $90 \mu \mathrm{L}$ of distilled water, and $10 \mu \mathrm{L}$ of freshly prepared ferric chloride $(0.1 \% \mathrm{w} / \mathrm{w})$ was added. The reducing power of each well was investigated through the transformation of $\mathrm{Fe}^{3+}$ to $\mathrm{Fe}^{2+}$, and its absorbance was measured at $700 \mathrm{~nm}$ using an ELx800 microplate reader. The ferric reducing antioxidant power (FRAP) values of plant extracts were derived from the calibration curve of ascorbic acid and expressed as the equivalent of ascorbic acid.

(4) DPPH Scavenging Method. The 2,2-diphenyl-1-picrylhydrazyl (DPPH) radical scavenging activity was determined using the method proposed by Ahmed et al. [30] adapted on 96-well microplates. A solution of $\mathrm{DPPH}$ $(100 \mu \mathrm{M})$ was freshly prepared by dissolving $3.9 \mathrm{mg} \mathrm{DPPH}$ in $100 \mathrm{~mL}$ of methanol. Aliquots of $180 \mu \mathrm{L}$ of DPPH solution were added to $20 \mu \mathrm{L}$ of varying concentrations (3-400 $\mu \mathrm{g} /$ $\mathrm{mL}$ ) of the extract or ascorbic acid. The microplate was then 
incubated in the dark for $30 \mathrm{~min}$ at room temperature. The blank was prepared as above mentioned without the extract replaced by methanol. Changes in the absorbance of the extract samples were measured at $517 \mathrm{~nm}$ using an ELx800 microplate reader. Radical scavenging activities (RSA) were expressed as the inhibition percentage calculated using the following formula:

$$
\operatorname{RSA}(\%)=\frac{\text { Absorbance }_{\mathrm{DPPH}}-\text { absorbance }_{\text {sample }}}{\text { Absorbance }_{\mathrm{DPPH}}} \times 100 \text {. }
$$

The extract concentration corresponding to $50 \%$ inhibition $\left(\mathrm{EC}_{50}\right)$ was calculated from the curve of inhibition percentage against the extract concentration.

2.5. Statistical Analysis. All assays were performed in triplicate. The data were subjected to analysis of variance, and when differences were observed, the means were compared two by two using the Waller-Duncan test $(p<0.05)$. Each dependent variable (yield, TPC, TFC, TfnC, TTC, TAC, FRAP, DPPH, and $\mathrm{IC}_{50}$ of $\alpha$-amylase and $\alpha$-glucosidase inhibition) was compared in the three groups of independent variables (phenological stage of $P$. capitata, host, and extraction solvent). The results were expressed as mean\pm standard deviation. Pearson correlation was used to search for the link between the different dependent variables at 5\% and 1\% thresholds. For these analyses, we used SPSS 25 software for Windows.

\section{Results}

3.1. Extraction Yields of Phragmanthera capitata. Extraction yields varied with the solvents used for extraction, the host plant type, and the phenological stage of $P$. capitata (Figure 1). Using methanol as the extracting solvent gave significantly higher $(p<0.05)$ yields compared to using either ethanol, water, ethyl acetate, or hexane, the latter giving the lowest yields. The extraction yield of $P$. capitata harvested on $P$. guajava and $P$. mannii was overall significantly higher $(p<0.05)$ during flowering for an extraction solvent except with hexane. On the other hand, the extraction yield of $P$. capitata from $P$. americana host was not significantly influenced by the phenological stage of $P$. capitata.

3.2. HPLC Profile of Ethyl Acetate Extracts. HPLC profiles of ethyl acetate extracts showed a dynamic accumulation of phenolic compounds in $P$. capitata according to the phenological stage and host plant (Figure 2). The chemical composition of the ethyl acetate extract of $P$. capitata grown on $P$. americana and $P$. mannii was relatively the same before and during flowering. Indeed, during the vegetative stage, quercetin appeared as the main constituent, while during flowering, rutin replaced quercetin as the main constituent. The main differences between these two plant hosts appear during fruiting. At this stage, while tannic acid was highly synthesized in replacement of rutin in P. capitata growing on $P$. americana, rutin remained the most abundant constituent on $P$. mannii. Ethyl acetate extract of $P$. capitata collected on $P$. guajava presented a relatively stable chemical composition at the three phenological stages, but very different from what was observed on $P$. americana and $P$. mannii. The main constituent of this extract was not identified.

3.3. In Vitro Inhibition of $\alpha$-amylase and $\alpha$-glucosidase Activities. The influence of the solvent, phenological stage, and host on $\alpha$-amylase and $\alpha$-glucosidase inhibition by various extracts is presented in Figure 3. Extract from $P$. capitata collected on $P$. americana and $P$. guajava presented higher inhibitory activities (low $\mathrm{IC}_{50}$ compared to acarbose whose dose-activity is showed in Supplementary material) at vegetative and fruiting stages. With P. mannii, this inhibitory activity increased significantly during flowering and more at fruiting (Figure 3(a)). Ethyl acetate, methanol, and hexane extracts had the best inhibitory activity against $\alpha$-amylase.

The hexane extract had poor inhibitory activity on $\alpha$-glucosidase. The ethanol extract had a comparable inhibitory activity to that of the methanol extract. In general, regardless of the host and phenological stage, methanol, ethanol, and water extracts exhibited better inhibitory activities on $\alpha$-glucosidase compared to the ethyl acetate extract.

3.4. Phenolic Contents of Phragmanthera capitata. The phenolics contains in extracts were determined using a standard calibration curve (Supplementary material). The evolution of the phenolic contents of $P$. capitata as a function of the solvent, host, and phenological stage is presented in Figure 4. The total phenol contents were significantly $(p<0.05)$ higher with ethyl acetate, followed by hexane at all stages of development of $P$. capitata and on all hosts (Figure 4(a)). Water was the worst solvent for extracting these compounds, while methanol and ethanol had globally comparable effects. With hexane and ethyl acetate, the total phenol contents increased significantly during flowering and during fruiting of $P$. capitata grown on $P$. americana and on P. guajava. With the other solvents, the general tendency was a decrease of the phenol content, the levels of total phenols being higher when $P$. capitata was harvested from P. mannii.

The total flavonoid contents of the extracts varied according to the extraction solvent, the phenological stage, and the host plant on which P. capitata grows (Figure 4(b)). Thus, this class of phenolic compounds was best extracted with methanol, ethanol, and ethyl acetate. Hexane and water extracted the lowest, regardless of the phenological stage and the host plant. However, for $P$. capitata collected on P. mannii, the total flavonoids were better extracted by ethyl acetate, and their contents were significantly different from those of methanol and ethanol extractions, respectively. Overall, the flavonoid levels of $P$. capitata were significantly higher during fruiting, especially when extracted with ethyl acetate and to a lesser extent ethanol and methanol regardless of the plant host on which $P$. capitata grows. 


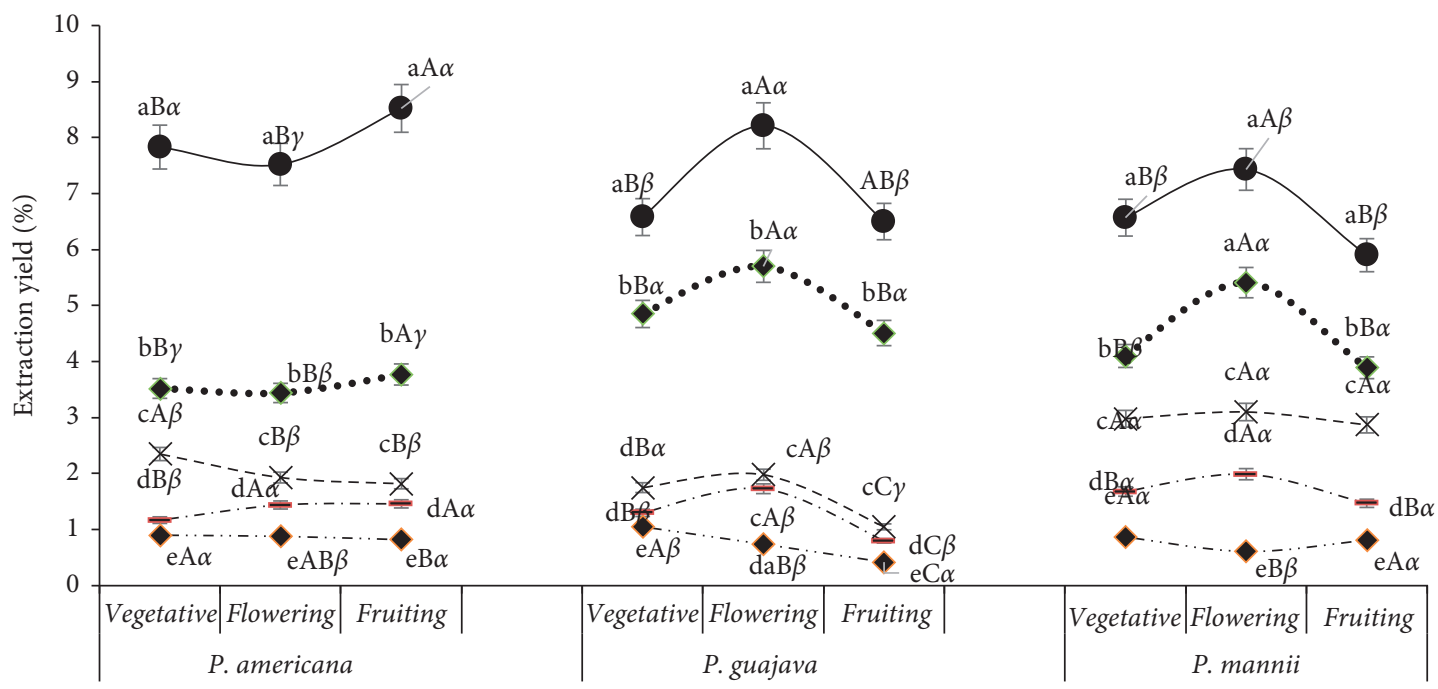

Phenological stage/host plant

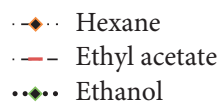

- -. Hexane
.-- Ethyl acetate

..... Ethanol

Figure 1: Evolution of the extraction yields of P. capitata according to the solvent, phenological stage, and host. a, b, c, d, e: for the same phenological stage and host plant, means carrying different letters are significantly different $(p<0.05$, Waller-Duncan test); A, B, C: for the same solvent and host plant, means carrying different letters are significantly different $(p<0.05$, Waller-Duncan test), $\alpha, \beta, \gamma$ : for the same solvent and host phenological stage, means carrying different letters are significantly different $(p<0.05$, Waller-Duncan test).

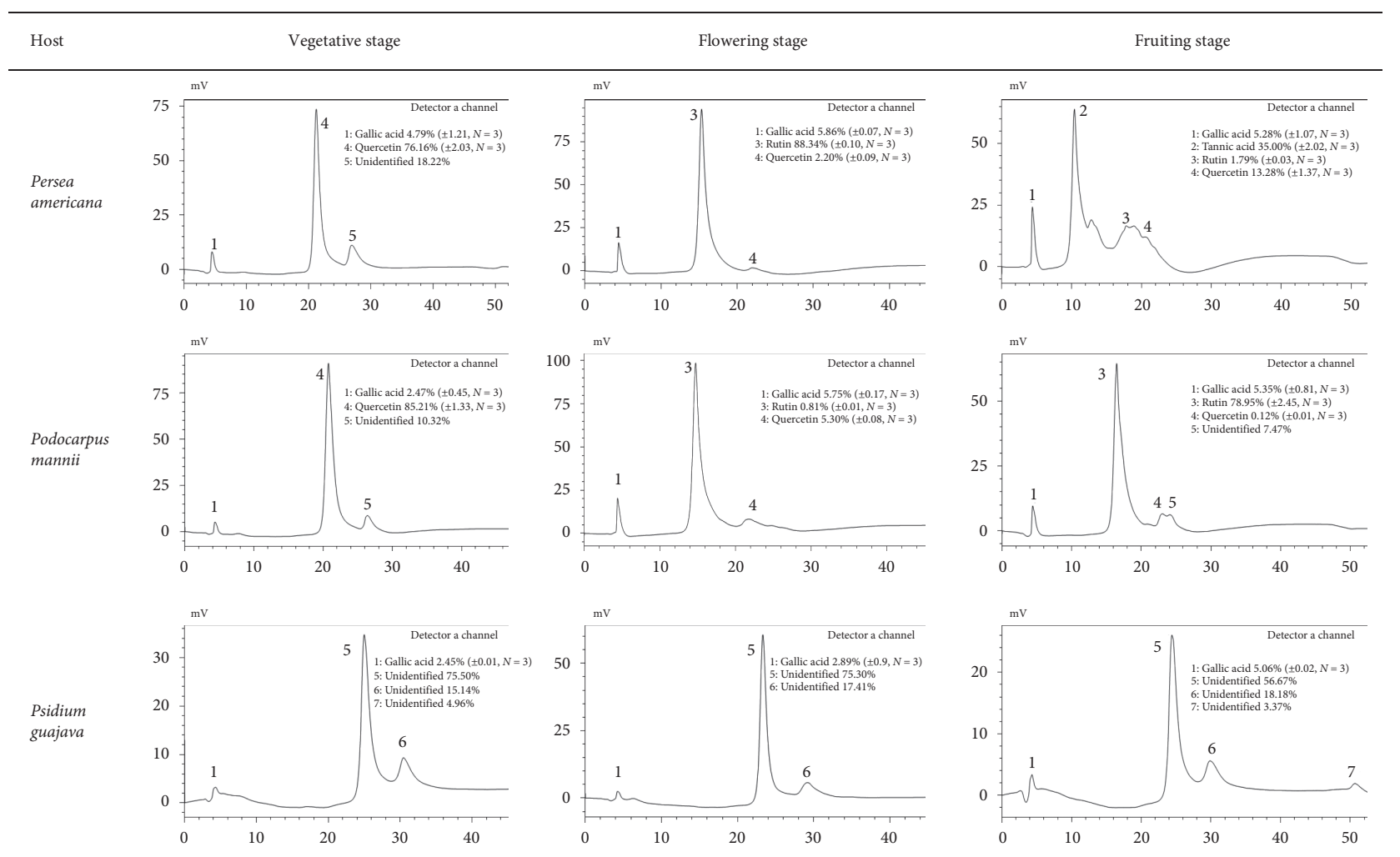

Figure 2: HPLC profile at $280 \mathrm{~nm}$ of the ethyl acetate extract of Phragmanthera capitata harvested from tree hosts at three different phenological stages. 


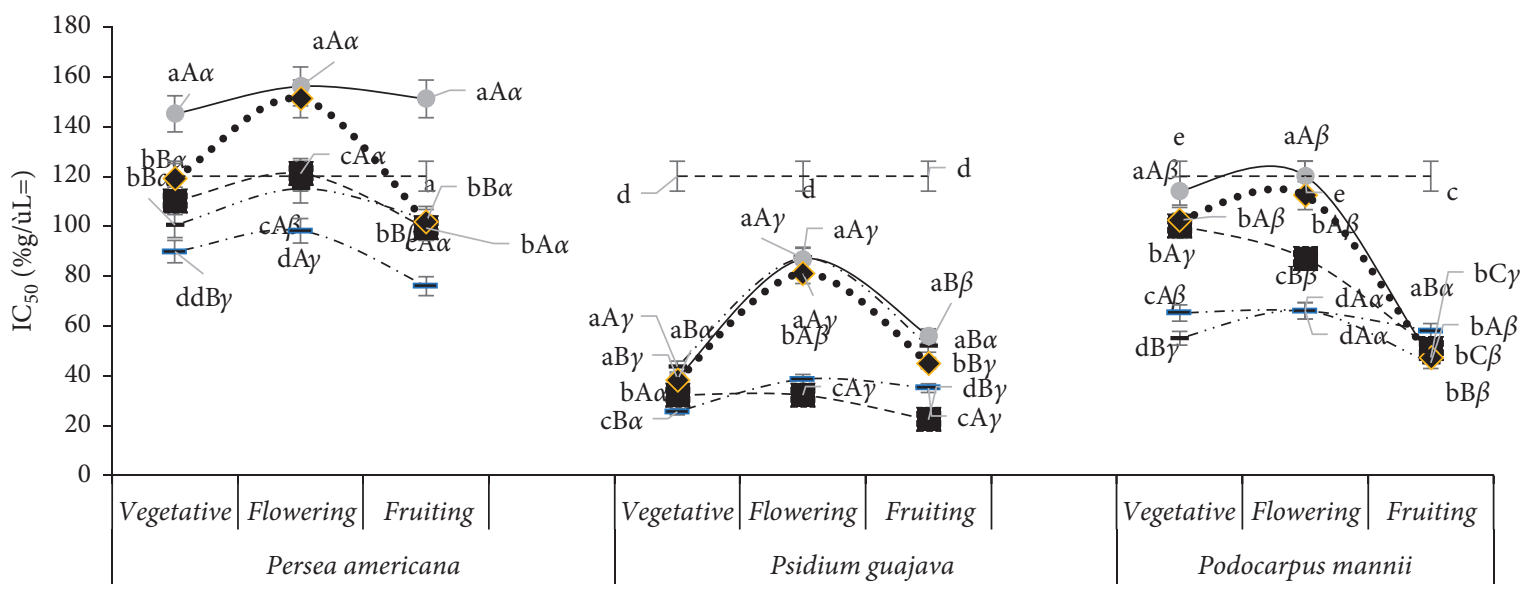

Phenological stage/host plant

$$
\begin{aligned}
& \text {-.. Hexane } \\
& \text { - Ethyl acetate } \\
& - \text { - Ethanol }
\end{aligned}
$$

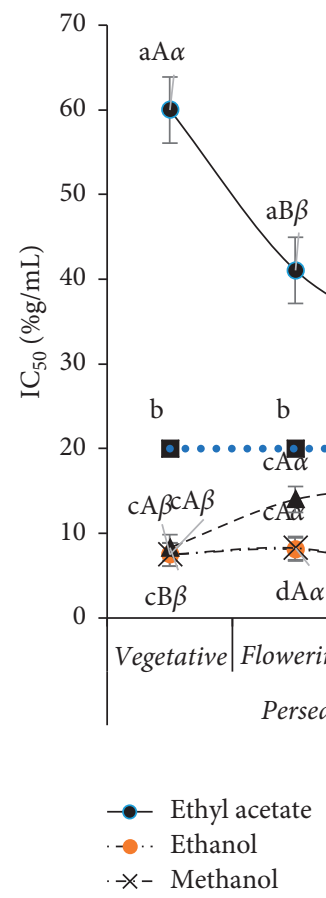

..... Methanol

- - Distilled water

-. - Acarbose

(a)$$
\text { . }
$$ 

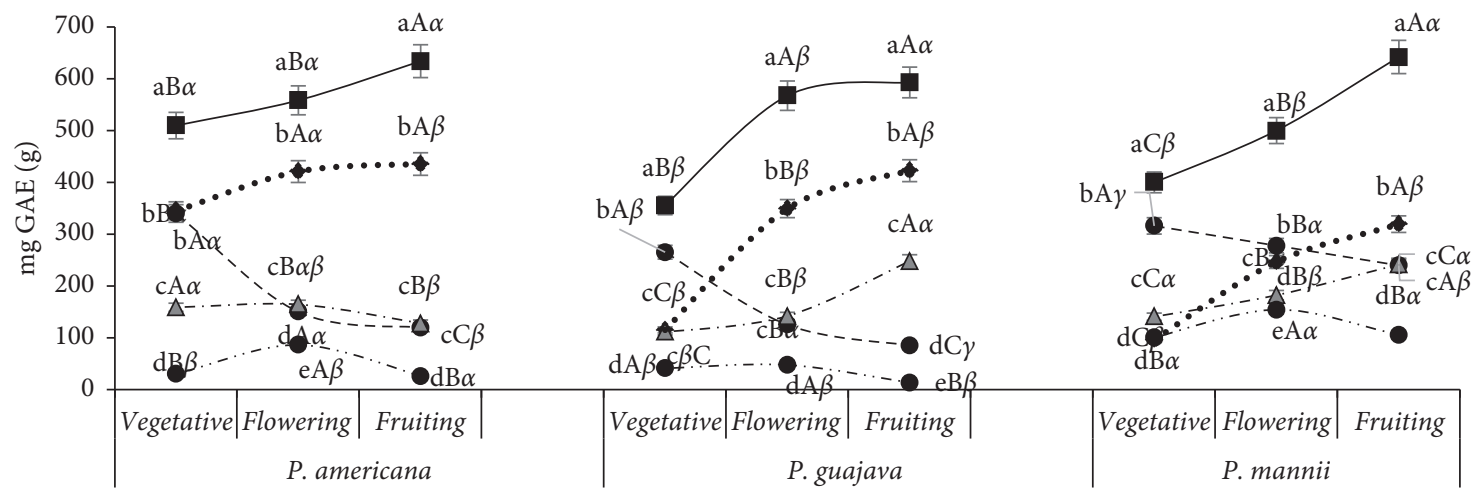

Phenological stage/host plant

$$
\begin{aligned}
& \cdots \bullet \text { Hexane } \\
& - \text { Ethyl acetate } \\
& -\bullet-\text { Ethanol }
\end{aligned}
$$

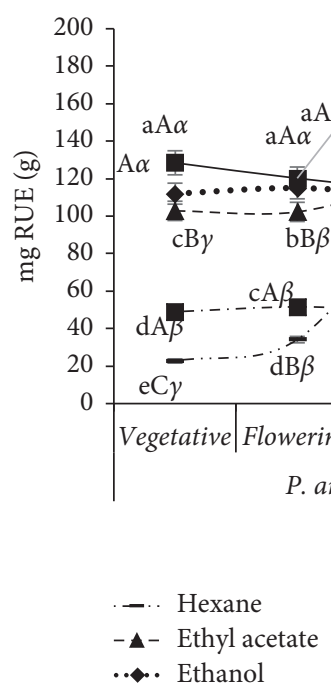

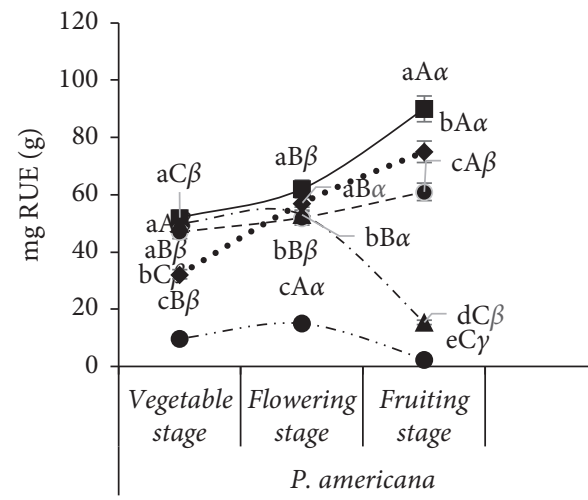

$-\Delta$ - Methanol

- Distilled water

(a)
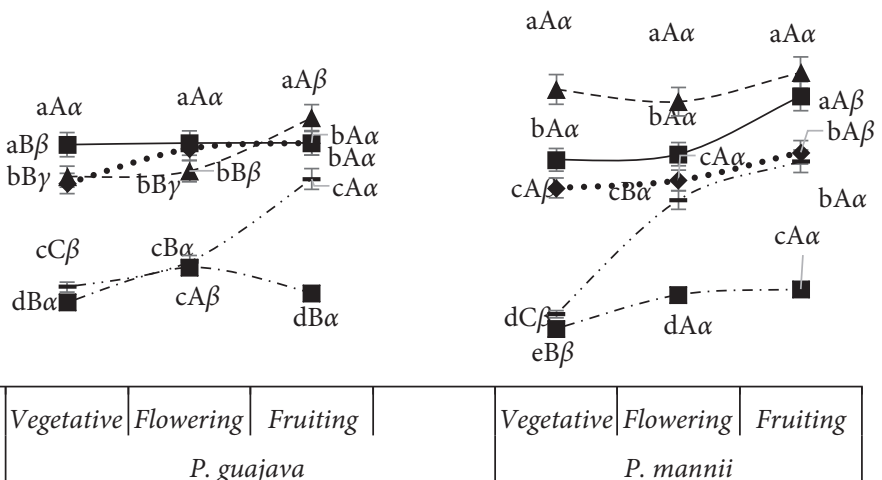

Penological stage/host plant

- Methanol

- Distilled water

(b)
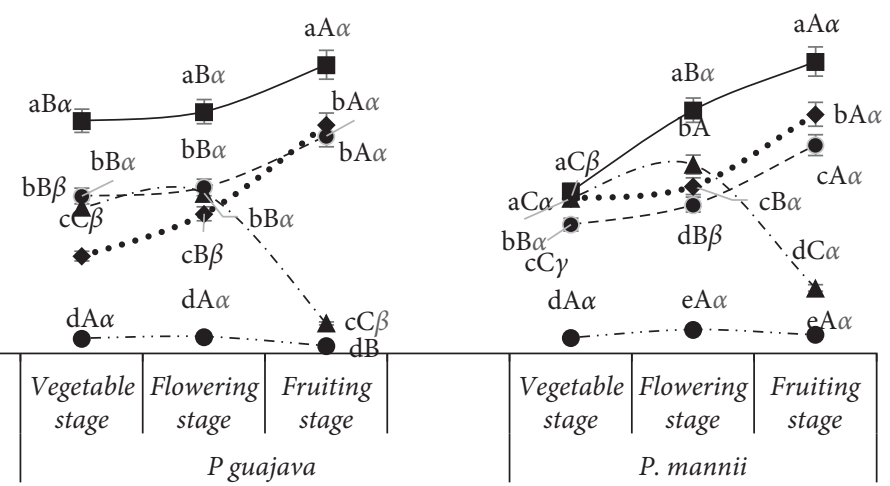

Phenological stage and host plant

-.. Hexane

$\rightarrow$ Ethyl acetate

.... Methanol

- - Distilled water

- •- Ethanol

Figure 4: Continued. 


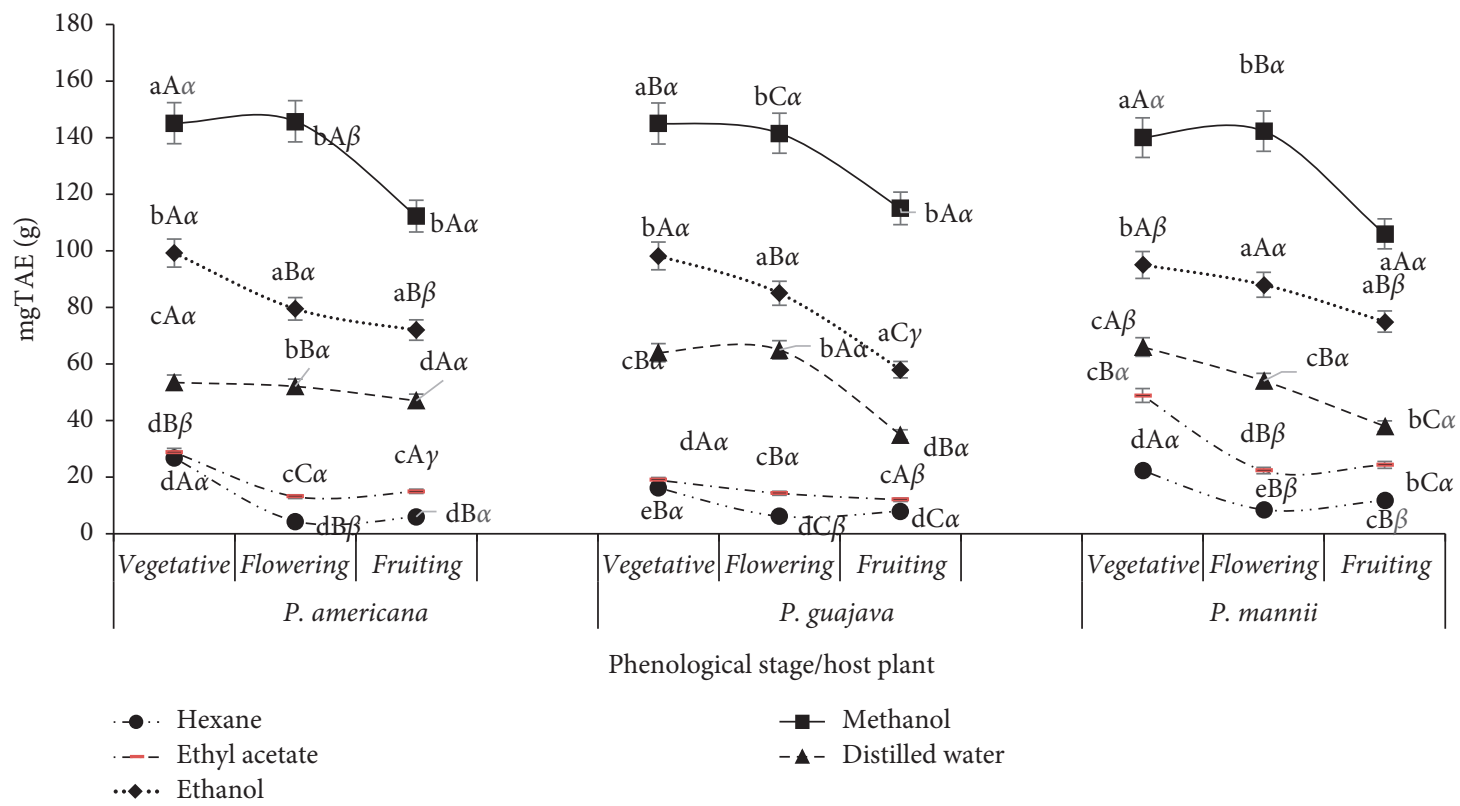

(d)

Figure 4: Evolution phenolic contents in Phragmanthera capitata extracts according to the phenological stage, host, and solvent of extraction. a, b, c, d, e: for the same phenological stage and host plant, means carrying different letters are significantly different $(p<0.05$, Waller-Duncan test); A, B, C: for the same solvent and host plant, means carrying different letters are significantly different $(p<0.05$, Waller-Duncan test), $\alpha, \beta, \gamma$ : for the same solvent and host phenological stage, means carrying different letters are significantly different ( $p<0.05$, Waller-Duncan test). (a) Total phenol contents. (b) Total flavonoid contents. (c) Total flavonol contents. (d) Total tannin contents.

water. With these three solvents, the rate of tannins decreased significantly during fruiting in all extracts, regardless of the host of P. capitata. With hexane and ethyl acetate, the tannin levels were low and decreased significantly during flowering except when the host was P. mannii, for which these levels remained constant during flowering and during fruiting.

3.5. Antioxidant Activities of Phragmanthera capitata Extracts. The total antioxidant capacity, DPPH scavenging, and FRAP activities in various extracts of $P$. capitata varied according to the extraction solvent, the phenological stage, and the host plant (Figure 5). The total antioxidant capacity of the P. capitata extracts by various solvents decreased significantly in the following order: ethanol and methanol, ethyl acetate and hexane, and water at all stages of development of the plant and plant host (Figure 5(a)). The total antioxidant capacity decreased significantly during flowering regardless of the host plant and the extraction solvent, even though that of the aqueous extract increased during flowering. Furthermore, with ethanol and methanol, the total antioxidant capacity was significantly higher before and during fruiting of P. capitata growing on P. guajava, followed by that of P. mannii.

The solvents that gave extracts with better reducing power, in the ascending order were ethanol, methanol, and water regardless of the phenological stage or the host plant (Figure 4). Hexane and ethyl acetate gave extracts with worst reducing power (Figure 5(b)). For all solvents, the general trend was a decrease in the reducing power during flowering of $P$. capitata regardless of the host plant. On the other hand, with ethanol and methanol, the reducing power was higher before and during fruiting of $P$. capitata growing on P. guajava and on P. mannii.

Ethanol and methanol provided extracts with stronger DPPH scavenging activity, close to that of ascorbic acid regardless of the phenological stage and the host plant (Figure 5(c)). With hexane and ethyl acetate, the P. capitata extract had worst DPPH scavenging activity, and this activity was significantly weaker at the flowering stage of the plant. With water, DPPH scavenging activity of $P$. capitata extracts was intermediate to that of the extracts with solvents ethanol/methanol and ethyl acetate before and during flowering and significantly weaker during fruiting for all host plants. On the other hand, for all extracting solvents, DPPH scavenging activity of $P$. capitata extracts was not influenced by the host plant.

\section{Discussion}

The multiple biological activities and traditional use of $P$. capitata make this plant a good candidate for the preparation of a multiusage phytomedicine, especially against diabetes and oxidative stress, one of the factors that play a great role in the pathogenesis of diabetes [31]. However, for this to be effective, it is important to define the minimum conditions of its collection and preparation to guarantee its effectiveness at all times. This action goes through standardization to fulfill some basic requirements to guarantee phytomedicine efficacy, safety, and reproducibility. In this 


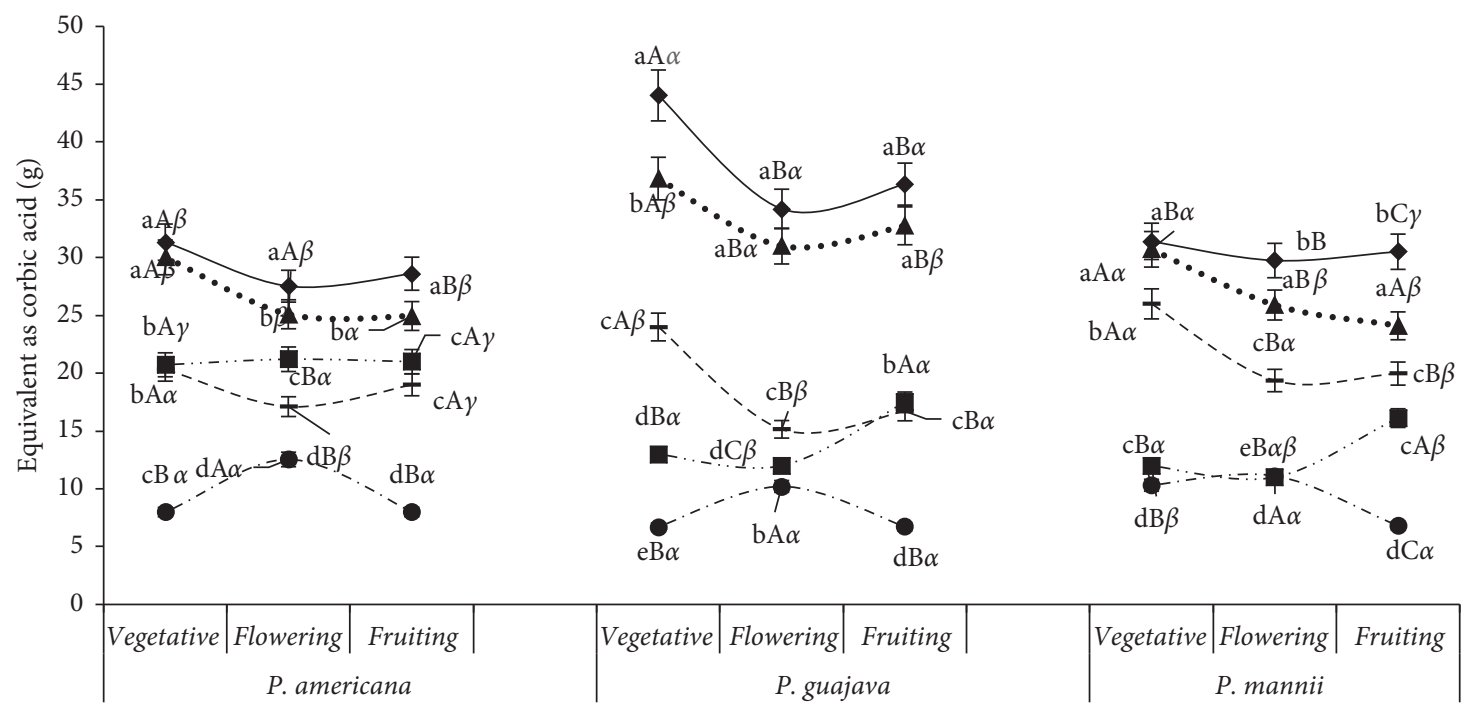

Phenological stage/host plant

-. Hexane

Ethyl acetate

$\multimap$ Ethanol

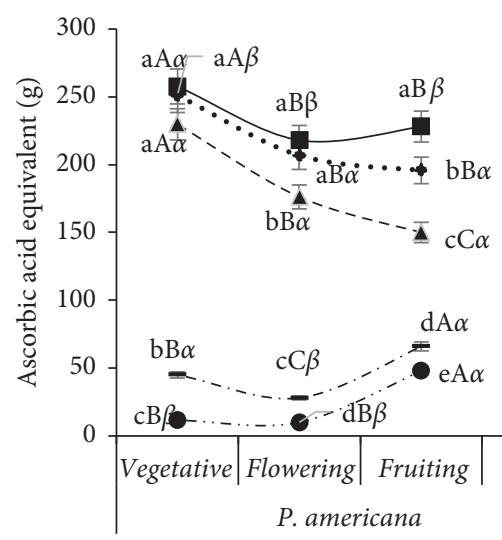

-. Hexane

-- Ethyl acetate

- Ethanol
..A.. Methanol

- Distilled water

(a)
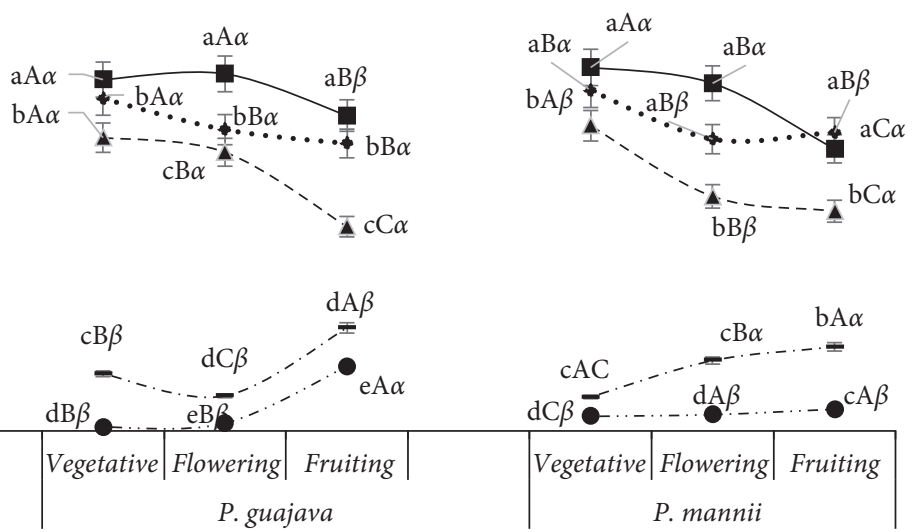

Phenological stage and host plant

..*. Methanol

- - Distilled water

(b)

Figure 5: Continued. 

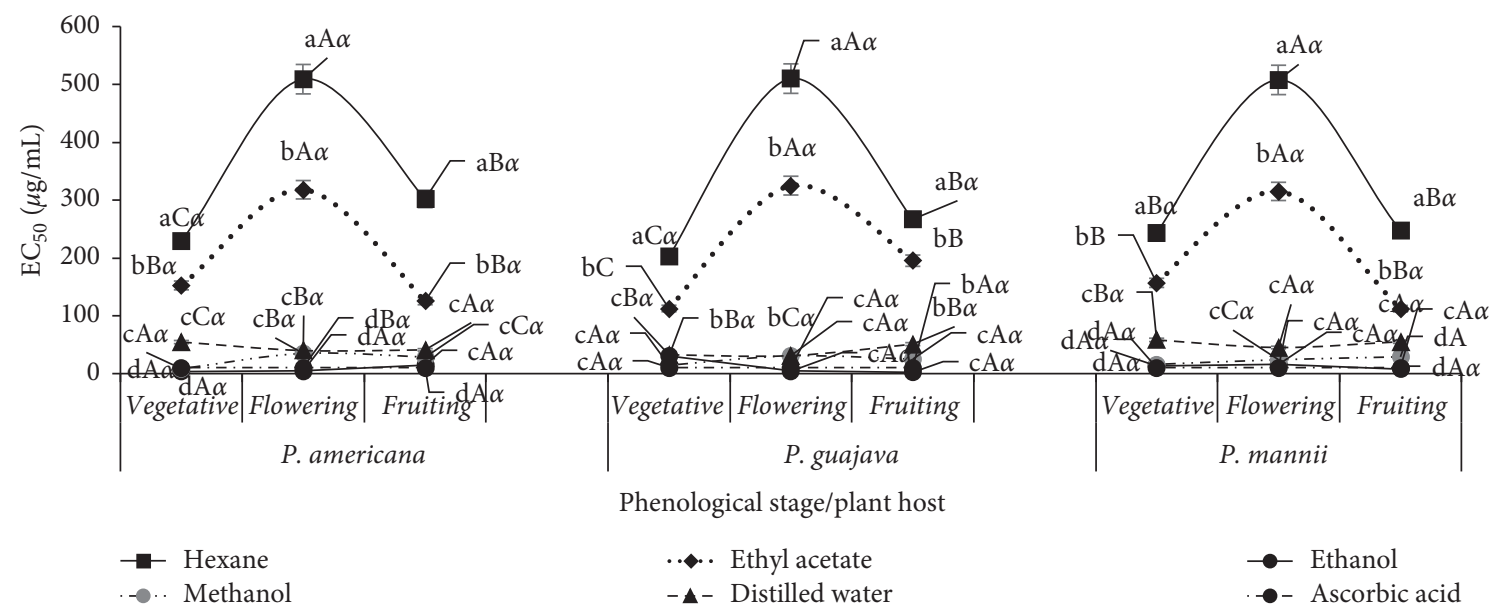

(c)

Figure 5: Evolution of antioxidant capacities of Phragmanthera capitata extracts according to the phenological stage, host, and solvent of extraction. a, b, c, d, e: for the same phenological stage and host plant, means carrying different letters are significantly different $(p<0.05$, Waller-Duncan test); A, B, C: for the same solvent and host plant, means carrying different letters are significantly different $(p<0.05$, Waller-Duncan test), $\alpha, \beta, \gamma$ : for the same solvent and host phenological stage, means carrying different letters are significantly different ( $p<0.05$, Waller-Duncan test). (a) Total antioxidant capacities. (b) FRAP activities. (c) DPPH.

light, we studied some parameters that could influence the antidiabetic and antioxidant properties, the extraction yield, and the chemical profile of $P$. capitata extracts, including the nature of the extraction solvent, the host plant, and the phenological stage of $P$. capitata and observed that these factors had more or less marked effects on the studied properties.

Solid-liquid (plant powder-solvent) extraction was used in this study, for which the constituents of the plant are transferred to the solvent with a speed and efficiency which depended on the polarity, viscosity, and corrosive properties of the extracting solvent $[30,32]$. In this light, the extraction yield of $P$. capitata was higher with polar solvents, namely, water, methanol, and ethanol, methanol being the best. Methanol, an amphiphilic solvent, with low viscosity and density, has the ability to weaken cellular structures, penetrate, and diffuse into the plant cell to solubilize a large range of molecules in the plant cells, mostly polar ones but also a large portion of nonpolar [31-34]. These properties may justify why this solvent gave better extraction yields than water. Our results are in accordance with those obtained for P. capitata growing on rubber in Nigeria [17].

Phenolic compounds belong to a large family of secondary metabolites and possess different chemical structures and polarities and are differently extracted by various solvents [35]. The difference in extractive capacities of solvents on phenolic compounds of $P$. capitata is in agreement with the findings of Ohikhena et al. [17] and Freitas et al. [36]. The global trend was a decrease in the total phenol, flavonoid, and flavonol contents with the increase of solvent polarity, particularly with methanol. For this solvent, the extraction yield was significantly and negatively correlated to TPC $(r=0.877, p<0.01)$, TFC $(r=-0504, p<0.01)$, and TFnC $(r=-701, p<0.01)$, while TTC was positively correlated to the extraction yield $(r=0.572, p<0.01)$ by this solvent
(Table 2). This can be justified by the fact that, in methanol extracts, these compounds were surely diluted, due to the high and nonspecific extractive capacity of plant secondary metabolites by methanol, allowing it to extract a large variety of compounds, both polar and nonpolar [37]. The positive correlation of the yield to TTC indicates that tannins were more efficiently extracted by methanol. Indeed, tannins are polymerized into complex forms of phenolic compounds with increased polarity [38]. The yield was positively correlated to TFC $(r=0.868 ; p<0.01)$ and TTC $(r=0.529$; $p<0.05$ ) of ethyl acetate extract (Table 2), suggesting that theses extracts mostly contain aglycone flavonoids. It is known that less polar solvents such as ethyl acetate extract preferably extract aglycone flavonoids, while more polar solvents such as alcohol and water better extract glycoside flavonoids [39]. These correlations, associated to one of the methanol extractions, may indicate that the total tannins of $P$. capitata are midpolar compounds. However, it is to be noted that many authors recommend solvent mixtures for tannin extraction [40], and this may later be experimented for $P$. capitata tannin extraction, combining, for example, methanol and ethyl acetate in appropriate proportions.

Plants contain a wide range of active principle described as having hypoglycemic effects, mainly glycosides, alkaloids, hypoglycans, galactomannan gum, polysaccharides, steroids, peptidoglycan, guanidine, glycol peptides, and terpenoids [41]. Some of these principles are $\alpha$-amylase inhibitors, and many studies have focused their attention on phenolic compounds as amylase inhibitors [42] and as antioxidant [43]. $\alpha$-amylase and $\alpha$-glucosidase are a key enzyme in digestion of starch, and as such, their inhibitors such as acarbose are used to control postprandial glycemic in type- 2 diabetic [44]. Recent studies show a renewed interest in plant phenolic compounds as inhibitors of $\alpha$-amylase and $\alpha$-glucosidase [45]. In the present study, the inhibition of 
TABle 2: Significant Pearson correlation coefficients between yield and $\alpha$-amylase and $\alpha$-glucosidase inhibition, phenolic contents, and antioxidant activities of various extracts from Phragmanthera capitata as a function of solvent of extraction (regardless the host plant).

\begin{tabular}{|c|c|c|c|c|c|c|c|}
\hline \multicolumn{5}{|c|}{ Phenolic contents } & \multirow{2}{*}{$\begin{array}{c}\text { Antioxidant properties } \\
\text { FRAP }\end{array}$} & \multicolumn{2}{|c|}{ Enzyme inhibition } \\
\hline Extracts & TPC & TFC & TfnC & TTC & & $\alpha$-amylase & $\alpha$-glucosidase \\
\hline Hexane & & $-0.573^{* *}$ & & $0.385^{*}$ & $-0.558^{* *}$ & $-0.452^{*}$ & \\
\hline Ethyl acetate & & $0.868^{* *}$ & & $0.529^{* *}$ & & & \\
\hline Ethanol & & & & & $0.384^{*}$ & $-0.497^{* *}$ & \\
\hline Methanol & $-0.877^{* *}$ & $-0.504^{* *}$ & $-0.701^{* *}$ & $0.572^{* *}$ & & $-0.487^{*}$ & $0.485^{*}$ \\
\hline Aqueous & $0.645^{* *}$ & & & & & & \\
\hline
\end{tabular}

${ }^{*} p<0.05 .{ }^{* *} p<0.01$.

$\alpha$-amylase was negatively correlated to yield for hexane $(r=-0.452, p<0.05)$, ethanol $(r=-0.497, p<0.01)$, and methanol $(r=-0.487, p<0.01)$ extracts, indicating that high yield is associated to low $\mathrm{IC}_{50}$ and consequently high inhibition (Table 2). The inhibitory capacity of the hexane extract was not correlated to any phenolic content, a suggestion that the active principles in these extracts are not phenolic compounds. This was not the case with glucosidase that reveals a positive correlation between extraction yield and enzyme inhibition for methanol extracts $(r=0.485$, $p<0.05)$. In contrast, for this extract, a negative correlation was observed between $\mathrm{IC}_{50}$ of $\alpha$-glucosidase $(r=-0.694$, $p<0.01$ ) and TfnC (Table 3 ). For the ethyl acetate extracts, the inhibitions of $\alpha$-amylase and $\alpha$-glucosidase were negatively correlated with TPC $(r=-0.433, p<0.05)$ and TfnC $(r=-0.496, p<0.01)$, suggesting that the active ingredients in these extracts could be flavonols. With ethanol extracts, the correlation between enzyme inhibition was negative for TFC $(r=-0.602, p<0.01)$ and TfnC $(-0,672, p<0.01)$, suggesting that the active principles could be distributed in these two groups of phenolic compounds. With the methanol extract, a negative correlation was highlighted between TPC $(r=-0.735, p<0.01)$ and TfnC $(r=-0.746, p<0.01)$ and the inhibition of $\alpha$-glucosidase, whereas for $\alpha$-amylase, this correlation exists with TTC $(r=-0.616, p<0.01)$. For the aqueous extract, no correlation was highlighted.

With ethanol and methanol extracts, inhibition of $\alpha$-amylase was positively correlated with FRAP $(r=0.486$ and 0.528 , respectively, $p<0.01$ ), showing that the active compounds could also have ferric reducing ability. According to Asgar [44], it is important to keep equilibrium between $\alpha$-amylase and $\alpha$-glucosidase inhibitors, which efficiently limits the gastrointestinal adverse effects related to undigested starch reaching the colon. P. capitata extract that inhibited both $\alpha$-amylase and $\alpha$-glucosidase (except hexane extract) responding to this need. With lower $\mathrm{IC}_{50 \mathrm{~s}}$, the activities of the extracts on $\alpha$-glucosidase were more pronounced, and this has varied with the extraction solvent. $\mathrm{IC}_{50}$ of methanol and ethyl acetate extracts were negatively correlated to these properties for TPC and TfnC. This shows that the compounds which inhibit $\alpha$-glucosidase are different from those which inhibit $\alpha$-amylase and are mainly effective in the flavonol group. P. capitata growing on rubber tree in Nigeria showed a weak $\alpha$-amylase inhibition across all the solvent extracts and stronger $\alpha$-glucosidase inhibition like our results [18]. The weak $\alpha$-amylase inhibition by $P$. capitata grown on rubber in the previous study compared to our results may show the influence of the host plant and, to a lesser extent, the geographical conditions, and thus the importance of standardization.

As a mistletoe, $P$. capitata is capable of penetrating the living tissue of the host plant's stems and branches to extract the necessary resources for their survival. For this purpose, this parasitic plant uses haustorium induced by host-derived phenolic compounds to penetrate host tissues [46]. It depends on its host for water, nutrients, and some carbon compounds. It is also suggested that active compounds may pass from the host trees to the parasitic plants [1, 47]. On the other hand, the parasitic plant is able to synthesize its own secondary metabolites to fight against the resistance of the host [48]. This bilateral relationship may justify the differences in composition of phenolic compounds, and therefore the difference in the inhibition of glycolytic enzymes observed about the three host plants, which have different physiological and photosynthetic behaviors, and they differently react to infestation by $P$. capitata. This is in accordance with the results of $[47,49]$ who showed that Viscum album (European mistletoe) exhibited different levels of total phenolic acids and antioxidant activity when parasitizing different host species. In addition, it is important to mention that Dibong et al. [50] showed that Persea americana, with $9.38 \%$ abundance and $21.51 \%$ parasitism, is more susceptible to $P$. capitata than $P$. guajava with $4.12 \%$ abundance and 35\% parasitism in Cameroun. This shows that the two plants possess two different mechanisms of defense that can justify the qualitative and quantitative differences in phenolic contents and activities of $P$. capitata collected on these two host plants.

The levels of secondary metabolites in plants can vary depending on the environmental conditions and the stage of development of the plant [51]. Phenolic compounds, which are one of the main groups of secondary metabolites, are not an exception. In the present study, it became clear that the total phenol contents increased significantly from the vegetative stage to the flowering stage and from the flowering stage to the fruiting stage. These results concur with those of Berezina et al. [52] who showed that the rate of production of phenolic compounds is lower in Vaccinium macrocarpon plants during budding compared to flowering and fruiting stages. The increase in the total phenolic content during flowering may be attributed to flavonoids, which are responsible for the coloring of pollen and petals of flowers and which in some cases represent up to $4 \%$ of the dry weight of these flowers [53], since, in the present study, the evolution 
TABle 3: Significant Pearson correlation coefficients between phenolic contents, antioxidant activities, and $\alpha$-amylase and $\alpha$-glucosidase inhibition according to extraction solvent (regardless the host plant).

\begin{tabular}{|c|c|c|c|c|c|c|c|c|c|c|}
\hline \multirow[b]{2}{*}{ Solvent } & & \multicolumn{3}{|c|}{ Phenolic contents } & \multicolumn{3}{|c|}{ Antioxidant activities } & \multicolumn{3}{|c|}{ Enzyme inhibition } \\
\hline & & TPC & TFC & TfnC & TTC & TAC & FRAP & DPPH & Amylase & Glucosidase \\
\hline \multirow{2}{*}{ Hexane } & FRAP & $0.591^{* *}$ & $0.622^{* *}$ & $-0.571^{* *}$ & & $0.476^{*}$ & & & & \\
\hline & $\mathrm{DPPH}$ & & & $0.487^{* *}$ & $-0.658^{* *}$ & & & & & \\
\hline \multirow{4}{*}{ Ethyl acetate } & FRAP & $0.527^{* *}$ & & $0.714^{* *}$ & -0.374 & & & $-0.418^{*}$ & $-0.561^{* *}$ & $-0.506^{* *}$ \\
\hline & DPPH & & & & & $-0.608^{* *}$ & $-0.418^{*}$ & & & \\
\hline & Amylase & $-0.433^{*}$ & & $-0.771^{* *}$ & $0.827^{* *}$ & $0.591^{* *}$ & $-0.561^{* *}$ & & & $0.581^{* *}$ \\
\hline & Glucosidase & $-0.496^{* *}$ & & $-0.611^{* *}$ & $0.626^{* *}$ & $0.572^{* *}$ & $-0.506^{* *}$ & & $0.581^{* *}$ & \\
\hline \multirow{4}{*}{ Ethanol } & FRAP & $0.466^{*}$ & $-0.414^{*}$ & $-0.643^{* *}$ & $0.701^{* *}$ & $0.393^{*}$ & & & & $0.486^{*}$ \\
\hline & DPPH & & $-0.644^{* *}$ & & $0.450^{*}$ & $0.532^{* *}$ & & & & $0.815^{* *}$ \\
\hline & Amylase & $0.555^{* *}$ & $-0.602^{* *}$ & $-0.691^{* *}$ & $0.432^{*}$ & $-0.691^{* *}$ & & & & \\
\hline & Glucosidase & $0.466^{*}$ & $-0.672^{* *}$ & $-0.577^{* *}$ & $0.747^{* *}$ & $0.566^{* *}$ & $0.486^{*}$ & $0.815^{* *}$ & & \\
\hline \multirow{4}{*}{ Methanol } & FRAP & & 0.157 & $-0.709^{* *}$ & $0.517^{* *}$ & $0.603^{* *}$ & & $-0.854^{* *}$ & 0.104 & $0.528^{* *}$ \\
\hline & DPPH & & 0.045 & $0.630^{* *}$ & -0.360 & $-0.576^{* *}$ & $-0.854^{* *}$ & & -0.098 & $-0.462^{*}$ \\
\hline & Amylase & $0.658^{* *}$ & $0.454^{*}$ & $0.510^{* *}$ & $-0.616^{* *}$ & 0.009 & 0.104 & -0.098 & & $-0.694^{* *}$ \\
\hline & Glucosidase & $-0.735^{* *}$ & -0.232 & $-0.746^{* *}$ & $0.646^{* *}$ & $0.526^{* *}$ & $0.528^{* *}$ & $-0.462^{*}$ & $-0.694^{* *}$ & \\
\hline \multirow{4}{*}{ Water } & FRAP & & & $0.678^{* *}$ & $0.802^{* *}$ & & & & & \\
\hline & DPPH & & $-0.553^{* *}$ & & $-0.397^{*}$ & & & & & $-0.418^{*}$ \\
\hline & Amylase & & & & & & & & & $-0.573^{* *}$ \\
\hline & Glucosidase & & & & & & & $-0.418^{*}$ & $-0.573^{* *}$ & \\
\hline
\end{tabular}

${ }^{*} p<0.05 .{ }^{* *} p<0.01$.

of flavonoids levels followed that of total phenols with a few exceptions. This is in agreement with the results of Medini et al. [54] which revealed that the total phenol content of Limonium densiflorum increased at the flowering stage as compared to the vegetative one. Flowering and fruiting of $P$. capitata occur during the raining season. Thus, both the hosts and $P$. capitata are under favorable photosynthetic conditions (sunshine, abundance of minerals, and water) [51], boosting the rate of secondary metabolite biosynthesis in these plants [52]. At the same time, in this study, the rate of production of total tannins decreased from the vegetative phase to the fruiting stage, certainly passing to flowers. The main function of tannins is not only to ensure the protection of the plant against microbial pathogens, harmful insects, and other herbivores but also allow the reinforcement of plant tissues [55]. Plants rich in phenolics can also negatively modify the growth of neighboring plants by limiting the nitrogen supply. It can therefore be estimated that during the flowering of the fruiting body, this defense system in $P$. capitata works less intensely.

\section{Conclusion}

In this study, the effect of the extraction solvent, host plant, and phenological stage on phytochemical compounds, antioxidant, and antidiabetic activities of Phragmanthera capitata were investigated. Our results show that the extraction solvent, host, and phenological stages significantly affect the phenolic content, antioxidant, and antidiabetic activities of $P$. capitata. Out of the five solvents used for extraction of phytochemical compounds from the vegetable material, ethyl acetate was the most suitable solvent for optimum recovery of the phenolic, flavonoid, and flavonol compounds of $P$. capitata; while ethanol and methanol were the most suitable for tannins. Among host plants, the total phenolic, flavonoid, flavonol, and tannin contents of $P$. capitata extract from Persea americana and Psidium guajava were significantly higher than those of $P$. capitata extract from Podocarpus mannii. Better total antioxidant and antidiabetic activities were found with $P$. capitata growing on P. americana and Psidium guajava. Moreover, both phenolic compounds and antioxidant and antidiabetic activities of $P$. capitata appeared higher during flowering and fruiting. The HPLC profile of the ethyl acetate extract showed that it contains gallic acid, quercetin, rutin, and tannic acid, which could be related to the biological properties of the plant. The phytochemical composition and HPLC profile revealed the dynamic accumulation of phenolic compounds and established a basis for determining the best solvent and phenological stage for harvesting of the plant to improve the content of beneficial compounds in the phytomedicine from $P$. capitata.

\section{Abbreviations}

TPC: Total phenols content

TFC: Total flavonoids content

TfnC: Total flavonols content

TTC: Total tannins content

TAC: Total antioxidant capacity

RUE: Rutin equivalent

TAE: Tannic acid equivalent

AUC: Area under curve

DPPH: 2,2-Diphenyl-1-picrylhydrazyl

GAE: Gallic acid equivalent

FRAP: Ferric ion reducing antioxidant power

HPLC: High-performance liquid chromatography

DMSO: Dimethyl sulfoxide 
DNSA: 3,5-Dinitrosalicylic acid

RSA: Radical scavenging activity.

\section{Data Availability}

The data used to support this study are made available from the corresponding author upon request.

\section{Conflicts of Interest}

The authors declare that there are no conflicts of interest.

\section{Authors' Contributions}

Jules-Roger Kuiate designed the study and finalized the manuscript. Césaire Feudjio was the field investigator, contributed to the study design, and drafted the manuscript. Muhammed Arfat Yameen supervised the field work. Guy Sedar Singor Njateng, Muhammed Ahsan Khan, and Stephen Lacmata Tamekou contributed to the data analysis and to draft the manuscript. James D. Simo Mpetga helped in isolation and structure determination of compounds. All authors read and approved the final manuscript.

\section{Acknowledgments}

This work was supported by the TWAS- CIIT 2018 Fellowship (3240299467) and the University of Dschang and the Cameroonian Ministry of Higher Education. The authors are grateful to Dr. Prof. Jamshed IQBAL (COMSATS University of Information Technology, Abbottabad Campus, Pakistan) for his technical assistance. The authors express their gratitude to Prof Tume Christopher and Dr Mbulli Inoncent Ali, for agreeing to read and correct the language in this article.

\section{Supplementary Materials}

Dose activities of acarbose and calibration curves of standards for phenolics content determination. Isolated compounds from the ethanol extract of Phragmanthera capitata collected during fruiting on Persea americana. (Supplementary Materials)

\section{References}

[1] Y. Lim, R. Rajabalaya, S. Lee et al., "Parasitic mistletoes of the genera Scurrula and Viscum: from bench to bedside," Molecules, vol. 21, no. 8, pp. 1048-1134, 2016.

[2] J. Nazaruk and P. Orlikowski, "Phytochemical profile and therapeutic potential of Viscum albumL," Natural Product Research, vol. 30, no. 4, pp. 373-385, 2016.

[3] A. Koffi, F. Kouassi, S. N'Goran, and D. Soro, "Les Loranthaceae, parasites des arbres et arbustes:cas du département de Katiola, au nord de la Côte d'Ivoire," International Journal of Biological and Chemical Sciences, vol. 8, no. 6, p. 2552, 2014.

[4] S. Dibong, R. Mony, C. Ladoh, I. Boussin, and A. Amougou, "Parasitism evolution of Loranthaceae in the ndogbong chiefdom's orchard (Douala, Cameroon)," International Journal of Plant, Animal and Environmental Sciences, vol. 1, no. 3, pp. 261-269, 2011.
[5] C. F. Ladoh-Yemeda, D. Ndongo, and E. M. Tomedi, "Medicinal potentials of Phragmanthera capitata (sprengel) S . Balle used in the city of Douala (Cameroon)," Haya: The Saudi Journal of Life Sciences, vol. 4, no. 1, pp. 1-14, 2019.

[6] S. Dibong, N. Engone Obiang, N. Din et al., "Les Loranthaceae:un atout pour l'essor de la pharmacopée traditionnelle au Cameroun," International Journal of Biological and Chemical Sciences, vol. 3, no. 4, pp. 746-754, 2009.

[7] G. Etame-Loe, "Évaluation des activités antioxydante et antiinflammatoire de l'extrait aqueux de l'haustorium de Phragmanthera capitata (Sprengel) S. Balle (Loranthaceae) récolté sur Psidium guajava sur les rats femelles adultes de la souche wistar," The Journal of Animal and Plant Sciences, vol. 36, no. 3, pp. 5933-5941, 2018.

[8] L. P. Takem, B. A. Lawal, and K. G. Demekong, "Potentiating effect of Phragmanthera capitata extract in haematopoietic activities in Wistar rats," International Journal of Pharmacy and Pharmaceutical Sciences, vol. 2, no. 1, pp. 1-6, 2015.

[9] F. U. Ohikhena, O. A. Wintola, and A. J. Afolayan, "Evaluation of the antibacterial and antifungal properties of Phragmanthera capitata (sprengel) balle (Loranthaceae), a mistletoe growing on rubber tree, using the dilution techniques," The Scientific World Journal, vol. 2017, Article ID 9658598, 8 pages, 2017.

[10] P. M. U. Takem and C. F. Poh, "Anti-secretory, gastroprotective and anti-ulcer activities of aqueous extract of Phragmanthera capitata S. Balle in rats," International Journal of Pharmaceutical Sciences and Research, vol. 5, no. 8, pp. 3560-3565, 2014.

[11] L. P. Takem, C. F. Poh, E. L. Kechi, and N. P. Abe, "Steroidogenetic and spermatogenetic activities of aqueous extract of Phragmanthera capitata in Wistar Rats," International Journal of Pharma Sciences, vol. 5, no. 10, pp. 609-614, 2014.

[12] C. Feudjio, G. Sedar, S. Njateng, and J. Kuiate, "Evaluation of antidiabetic activity of aqueous extract of leaves from Phragmanthera capitata (Sprengel) S. Balle (Laurenthaceae) in Wistar albino Rats," Journal of Diseases and Medicinal Plants, vol. 4, no. 4, pp. 96-109, 2018.

[13] B. C. Obi, Z. Igweze, V. Nwaogu, C. Ben, and T. C. Akunne, "Studies on the anticonvulsant and sedative effects of Jatropha curcas (Euphorbiaceae) and Phragmanthera capitata (Loranthaceae) in mice," Drug Discovery Today, vol. 13, pp. 95$102,2019$.

[14] F. U. Ohikhena, O. A. Wintola, and A. J. Afolayan, “Toxicity assessment of different solvent extracts of the medicinal plant, Phragmanthera capitata (Sprengel) Balle on Brine Shrimp (Artemia salina)," International Journal of Pharmacology, vol. 12, no. 7, pp. 701-710, 2016.

[15] A. Cimmino, M. Masi, D. Rubiales, A. Evidente, and M. Fernández-Aparicio, "Allelopathy for parasitic plant management," Natural Product Communications, vol. 13, no. 3, p. 294, 2018.

[16] A. K. Sachan, G. Vishnoi, and R. Kumar, "Need of standardization of herbal medicines in modern era," International Journal of Phytomedicine, vol. 8, no. 3, pp. 300-307, 2016.

[17] F. U. Ohikhena, O. A. Wintola, and A. J. Afolayan, "Quantitative phytochemical constituents and antioxidant activities of the mistletoe, Phragmanthera capitata (Sprengel) Balle extracted with different Solvents," Pharmacognosy Research, vol. 10, no. 1, pp. 16-23, 2018.

[18] F. U. Ohikhena, O. A. Wintola, and A. J. Afolayan, "Investigating the antidiabetic potential of Phragmanthera capitata, a mistletoe harvested from rubber tree," Journal of Herbs, Spices \& Medicinal Plants, vol. 24, no. 2, pp. 151-159, 2018. 
[19] T. M. Babu, C. K. Kumar, A. Vijayalakshmi, and V. Narasimha, "'Standardization of herbal drugs - a review," International Ayurvedic Medical Journal, vol. 5, no. 1, pp. 269-277, 2017.

[20] The World Health Organization (WHO), The World Conservation Union (iucn) and World Wide Fund for Nature (wwf), Guidelines on 'the Conservation of Medicinal Plants, The International Union for Conservation of Nature and Natural Resources (IUCN), and WWF - World Wide Fund for Nature, Gland, Switzerland, 1993.

[21] M. I. Kazeem, J. O. Adamson, and I. A. Ogunwande, "Modes of inhibition of $\alpha$-amylase and $\alpha$-glucosidase by aqueous extract of Morinda lucidaBenth leaf," BioMed Research International, vol. 2013, Article ID 527570, 6 pages, 2013.

[22] K. Bljajić, N. Šoštarić, R. Petlevski et al., "Effect of Betula pendula leaf extract on $\alpha$-Glucosidase and glutathione level in glucose-induced oxidative stress," Evidence-Based Complementary and Alternative Medicine, vol. 2016, Article ID 8429398, 8 pages, 2016.

[23] P. Kuppusamy, K. D. Lee, C. E. Song et al., "Quantification of major phenolic and flavonoid markers in forage crop Lolium multiflorum using HPLC-DAD," Revista Brasileira de Farmacognosia, vol. 28, no. 3, pp. 282-288, 2018.

[24] A. Horszwald and W. Andlauer, "Characterisation of bioactive compounds in berry juices by traditional photometric and modern microplate methods," Journal of Berry Research, vol. 1, no. 4, pp. 189-199, 2011.

[25] D. Laloo and A. N. Sahu, "Antioxidant activities of three Indian commercially available Nagakesar: an in vitro study," Journal of Chemical and Pharmaceutical Research, vol. 3, no. 1, pp. 277-283, 2011.

[26] F. M. Awah, P. N. Uzoegwu, P. Ifeonu et al., "Free radical scavenging activity, phenolic contents and cytotoxicity of selected Nigerian medicinal plants," Food Chemistry, vol. 131, no. 4, pp. 1279-1286, 2012.

[27] C. Gupta and R. Verma, "Visual estimation and spectrophotometric determination of tannin," Journal of Pharmaceutical Sciences and Research, vol. 2, no. 1, pp. 189-196, 2011.

[28] M. A. Hossain and M. D. Shah, "A study on the total phenols content and antioxidant activity of essential oil and different solvent extracts of endemic plant Merremia borneensis," Arabian Journal of Chemistry, vol. 8, no. 1, pp. 66-71, 2011.

[29] M. Vijayalakshmi and K. Ruckmani, "Ferric reducing antioxidant power assay in plant extract," Bangladesh Journal of Pharmacology, vol. 11, no. 3, pp. 570-572, 2016.

[30] D. Ahmed, K. Fatima, and R. Saeed, "Analysis of phenolic and flavonoid contents, and the anti-oxidative potential and lipid peroxidation inhibitory activity of methanolic extract of Carissa opaca roots and its fractions in different solvents," Antioxidants, vol. 3, no. 4, pp. 671-683, 2014.

[31] U. Asmat, K. Abad, and K. Ismail, "Diabetes mellitus and oxidative stress-A concise review," Saudi Pharmaceutical Journal, vol. 24, no. 5, pp. 547-553, 2016.

[32] F. Medini, H. Fellah, R. Ksouri, and C. Abdelly, "Total phenolic, flavonoid and tannin contents and antioxidant and antimicrobial activities of organic extracts of shoots of the plant Limonium delicatulum," Journal of Taibah University for Science, vol. 8, no. 3, pp. 216-224, 2014.

[33] S. Atun, S. Handayani, A. Rakhmawati, N. Aini Purnamaningsih, B. I. An Naila, and A. Lestari, "Study of potential phenolic compounds from stems of Dendrophthoe falcata (Loranthaceae) plant as antioxidant and antimicrobial agents," Oriental Journal of Chemistry, vol. 34, no. 5, pp. 2342-2349, 2018.
[34] F. Mohd, R. Abdul, K. Y. Pin, M. S. Zamree, and C. A. Luqman, "The effects of varying solvent polarity on extraction yield of Orthosiphon stamineus leaves," Journal of Applied Sciences, vol. 12, no. 11, pp. 1207-1210, 2012.

[35] A. Thouri, H. Chahdoura, A. El Arem, A. Omri Hichri, R. Ben Hassin, and L. Achour, "Effect of solvents extraction on phytochemical components and biological activities of Tunisian date seeds (var. Korkobbi and rechti)," $B M C$ Complementary and Alternative Medicine, vol. 17, p. 248, 2017.

[36] N. A. F. Fernandes, L. I. N. Canelo, R. da Conceição dos Santos Mata, and D. Mendonça, "In vitro antioxidant activity and total phenolic content of root extracts from Phragmanthera glaucocarpa (Peyr.) Balle," Journal of Natural Pharmaceutical Products, vol. 13, no. 4, pp. 1-7, 2018.

[37] Q. D. Do, A. E. Angkawijaya, P. L. Tran-Nguyen et al., "Effect of extraction solvent on total phenol content, total flavonoid content, and antioxidant activity of Limnophila aromatica," Journal of Food and Drug Analysis, vol. 22, no. 3, pp. 296-302, 2014.

[38] N. Junka, C. Rattanamechaiskul, C. Wongs-Aree, and S. Kanlayanarat, "Comparative study of organic solvents and extraction conditions on colour and antioxidant capacity in red cabbage," International Food Research Journal, vol. 24, no. 2, pp. 518-524, 2017.

[39] O. Ferreira and S. P. Pinho, "Solubility of flavonoids in pure solvents," Industrial \& Engineering Chemistry Research, vol. 51, no. 18, pp. 6586-6590, 2012.

[40] P. L. de Hoyos-Martínez, J. Merle, J. Labidi, F. Charrier, and El Bouhtoury, "Tannins extraction: a key point for their valorization and cleaner production," Journal of Cleaner Production, vol. 206, pp. 1138-1155, 2019.

[41] S. R. Mentreddy, "Medicinal plant species with potential antidiabetic properties," Journal of the Science of Food and Agriculture, vol. 87, no. 5, pp. 743-750, 2007.

[42] P. M. Sales, P. M. Souza, L. A. Simeoni, P. O. Magalhães, and D. Silveira, " $\alpha$-Amylase inhibitors: a review of raw material and isolated compounds from plant source," Journal of Pharmacy \& Pharmaceutical Sciences, vol. 15, no. 1, pp. 141183, 2012.

[43] B. S. Shah, L. Sartaj, F. Ali, A. S. I. Shah, and T. M. Khan, "Plant extracts are the potential inhibitors of $\alpha$-amylase: a review," MOJ Bioequivalence Bioavailab, vol. 5, no. 5, pp. 270-273, 2018.

[44] A. Asgar, "“Anti-diabetic potential of phenolic compounds : a review," International Journal of Food Properties, vol. 16, no. 1, pp. 91-103, 2013.

[45] R. Vinayagam, M. Jayachandran, and B. Xu, "Antidiabetic effects of simple phenolic acids: a comprehensive review," Phytotherapy Research, vol. 30, no. 2, pp. 184-199, 2016.

[46] Fotso, H. D. Mbouobda, M. A. Tita, R. F. Muyang, N. D. Belfiang, and N. D. Omokolo, "Parasitism of plum tree (Dacryodes edulis, burseraceae) by Loranthaceae in the locality of fotetsa-Dschang (west-Cameroon)," African Journal of Agricultural Research, vol. 9, no. 29, pp. 2255-2262, 2014.

[47] S. I. Vicaş, D. RuginǍ, L. Leopold, A. Pintea, and C. Socaciu, "HPLC Fingerprint of bioactive compounds and antioxidant activities of Viscum album from different host trees," Notulae Botanicae Horti Agrobotanici Cluj-Napoca, vol. 39, no. 1, pp. 48-57, 2011.

[48] M. Abualhasan, N. Jaradat, N. Abu-Hasan et al., "Bioactivity of Viscum album extracts from Olive and Almond host plants in Palestine," Pharmacognosy Journal, vol. 6, no. 2, pp. 38-44, 2014. 
[49] M. Luczkiewicz, W. Cisowski, P. Kaiser, R. Ochocka, and A. Piotrowski, "Comparative analysis of phenolic acids in mistletoe plants from various hosts," Acta Poloniae Pharmaceutica - Drug Research, vol. 58, no. 5, pp. 373-379, 2001.

[50] D. S. Dibong, D. Ndongo, P. R. Jules, T. V. Desiré, S. Georges, and A. Akoa, "Parasitism of host trees by the Loranthaceae in the region of Douala (Cameroon)," African Journal of Environmental Science and Technology, vol. 2, no. 11, pp. 371-378, 2008.

[51] Y. Cao, S. Fang, X. Fu, X. Shang, and W. Yang, "Seasonal variation in phenolic compounds and antioxidant activity in leaves of cyclocarya paliurus (batal.) iljinskaja," Forests, vol. 10 , no. 8 , pp. $624-8,2019$.

[52] E. V. Berezina, A. A. Brilkina, and A. P. Veselov, "Content of phenolic compounds, ascorbic acid, and photosynthetic pigments in Vaccinium macrocarpon Ait. dependent on seasonal plant development stages and age (the example of introduction in Russia)," Scientia Horticulturae, vol. 218, pp. 139-146, 2017.

[53] F. M. L. Ferreyra, S. P. Rius, and P. Casati, "Flavonoids: biosynthesis, biological functions, and biotechnological applications," Frontiers in Plant Science, vol. 3, pp. 1-15, 2012.

[54] F. Medini, R. Ksouri, H. Falleh, W. Megdiche, N. Trabelsi, and C. Abdelly, "Effects of physiological stage and solvent on polyphenol composition, antioxidant and antimicrobial activities of Limonium densiflorum," Journal of Medicinal Plants Research, vol. 5, no. 31, pp. 6719-6730, 2011.

[55] J.-P. Salminen and M. Karonen, "Chemical ecology of tannins and other phenolics: we need a change in approach," Functional Ecology, vol. 25, no. 2, pp. 325-338, 2011. 